\title{
Heart Rate Characteristics and Clinical Signs in Neonatal Sepsis
}

\author{
M. PAMELA GRIFFIN, DOUGLAS E. LAKE, T. MICHAEL O'SHEA, AND J. RANDALL MOORMAN
}

\begin{abstract}
Departments of Pediatrics [M.P.G.], Internal Medicine [D.E.L., J.R.M.] and Molecular Physiology and Biological Physics [J.R.M.], Cardiovascular Research Center, University of Virginia Health System, Charlottesville, VA 22908; Department of Pediatrics [T.M.O.], Wake Forest School of Medicine, Winston-Salem, NC 27157; MedImmune Corporation [M.P.G.], Gaithersburg, MD 20878
\end{abstract}

\begin{abstract}
To test the hypothesis that heart rate characteristic (HRC) monitoring adds information to clinical signs of illness in diagnosing neonatal sepsis, we prospectively recorded clinical data and the HRC index in 76 episodes of proven sepsis and 80 episodes of clinical sepsis in 337 infants in the University of Virginia NICU more than $7 \mathrm{~d}$ old. We devised an illness severity score based on clinical findings and tests relevant to sepsis. Point scores were derived from coefficients of multivariable regression models, and we internally validated a total score. We determined relationships of the HRC index with individual clinical signs, laboratory tests, and the total score. We found highly significant correlations of the clinical score and individual clinical signs with the HRC index. The clinical score and HRC index added independent information in predicting sepsis, and were similar in clinical and proven sepsis. The clinical score and the HRC index rose before sepsis, and the HRC index rose first. We conclude that clinical signs of illness and HRC monitoring add independent information to one another in the diagnosis of neonatal sepsis. (Pediatr Res 61: 222-227, 2007)
\end{abstract}

A cute clinical deterioration of infants in the NICU occurs frequently, especially in those of very low birth weight $(<1500 \mathrm{~g}$, VLBW), and late-onset bacterial sepsis is a common cause (1). Early diagnosis, before obvious clinical signs of illness, is an important goal but is difficult to achieve. The clinical signs of neonatal sepsis are neither specific nor uniform. Fanaroff et al. in the NICHD Neonatal Research Network found that increasing apnea, feeding intolerance, abdominal distension or guaiac-positive stools, increased respiratory support, lethargy and hypotonia were the most common presenting signs of sepsis, but none were found to have high predictive accuracy (2).

Diagnosis of late-onset neonatal sepsis is difficult even when infants appear ill because blood cultures have a substan-

Received June 28, 2006; accepted September 11, 2006.

Correspondence: J. Randall Moorman, M.D., Department of Medicine, Box 801395, University of Virginia, Charlottesville, VA 22908; e-mail: rm3h@virginia.edu

Medical Predictive Science Corporation of Charlottesville, Virginia, has a license to market technology related to heart rate characteristics monitoring of newborn infants, supplied partial funding for this study, and aided in collection of data. The company played no role in the study design, analysis and interpretation of data, writing of the report, or the decision to submit the paper for publication. M.P.G., and J.R.M. have an equity share in this company.

Additional support was provided by the American Heart Association, Mid-Atlantic Research Affiliate; University of Virginia Children's Hospital Research Fund; Virginia's Center for Innovative Technology; and NIGMS-64640 from the National Institute of General Medical Sciences.

Presented in part at the Pediatric Academic Societies' Meeting, May 3-6, 2003, Seattle, WA.

DOI: 10.1203/01.pdr.0000252438.65759.af tial false-negative rate, especially when based on small volumes of blood (3-7). Accordingly, the Centers for Disease Control recognizes "clinical sepsis" in infants, which requires only signs of illness with antibiotic therapy but no positive blood culture (8). The NICHD Neonatal Research Network found that both proven sepsis and clinical sepsis were associated with neurodevelopmental impairment in extremely low birth weight $(<1000 \mathrm{~g}$, ELBW) infants (9). The common denominator is the systemic inflammatory response syndrome (SIRS), the result of the host response to infectious and noninfectious insults (10). Clearly, new approaches to the early diagnosis of both proven and clinical neonatal sepsis are required.

Before physician suspicion of sepsis and SIRS, neonates have reduced heart rate variability and transient decelerations similar to the findings in distressed fetuses $(11,12)$. Heart rate measures optimized to detect these abnormal heart rate characteristics (HRC) $(11,13-15)$ were used to develop an HRC index at one NICU and then validated at another as a predictor of neonatal infection and death (16-18).

Laboratory tests such as the I:T ratio add predictive information independent of the HRC index (19). Clinicians also have ready access to many other sources of information about the status of the infant such as the general appearance, vital signs, apnea, oxygen requirement, and feeding tolerance. Our goal in this work was to determine whether the HRC index added to the clinical information that is already available to physicians in the diagnosis of late-onset neonatal sepsis.

Accordingly, we developed a clinical illness score that was specifically relevant to the diagnosis of neonatal sepsis. The method for development followed that of the Richardson score (20) for neonatal mortality or prolonged ventilation. Thus armed, we examined the relationship between HRC and objective measures of clinical illness. The major hypothesis was that HRC monitoring and clinical signs provide independent information in the early diagnosis of late-onset clinical and proven neonatal sepsis.

\section{METHODS}

Patient population. We studied all admissions to the University of Virginia NICU from August 2001 to July 2003 who were 7 or more days of age and had 7 or more days of HRC monitoring. The clinical research protocol

Abbreviations: HRC, heart rate characteristics; I:T ratio, ratio of immature to total neutrophils; VLBW, very low birth weight; WBC, white blood cell count 
was approved by the Human Investigation Committee of the University of Virginia.

Clinical and HRC index database. We prospectively recorded clinical signs and respiratory support into a relational events database (Microsoft Access), and laboratory results were available from an electronic archive. Each hospital course was divided into 6-h blocks beginning at midnight $(n=$ 41,769), and the HRC index and clinical score were noted for each block. Healthcare personnel were not aware of the result of the HRC monitoring.

The HRC index reflects the degree of reduced variability and transient decelerations in the prior $12 \mathrm{~h}$. We record RR intervals in sets of 4,096, approximately 20 to $25 \mathrm{~min}$ in duration, depending on the heart rate. For each we calculate SD, sample asymmetry (the characteristic abnormality is increased asymmetry due to more decelerations and fewer accelerations (13)) and sample entropy (SampEn, and the characteristic abnormality is a reduction due to outliers $(14,15,21))$. We select the median SD and sample asymmetry values over the preceding $12 \mathrm{~h}$, and the $10^{\text {th }}$ percentile lowest SampEn value. These are used in a regression expression relating these parameters to episodes of clinical illness that was developed at one center and validated at another (16). The result is the probability of clinical illness in the next $24 \mathrm{~h}$. It is divided by the average probability and presented as the fold-increase in risk of imminent clinical illness.

Proven sepsis and clinical sepsis events. We defined proven sepsis as clinical signs of sepsis and a positive blood culture prompting five or more days of antibiotic therapy. We defined clinical sepsis as clinical signs of sepsis with a negative blood culture prompting five or more days of antibiotic therapy (9). Recurrent episodes of sepsis were considered to be distinct if they occurred 7 or more days apart.

Clinical illness score. We (1) identified candidate findings of neonatal sepsis based on clinical experience, (2) made internally-validated regression models relating these findings to proven and clinical sepsis (22), and (3) used coefficients of the models to make an integer-based score (23).

The candidate findings that appeared in the final score were: Severe apnea requiring positive pressure ventilation or $50 \%$ increase in apneic episodes over $24 \mathrm{~h}$ in an extubated infant stable for three days; increased ventilatory support and $\mathrm{F}_{\mathrm{i}} \mathrm{O}_{2}$ by $25 \%$; temperature instability $\left(>38^{\circ} \mathrm{C}\right.$ or $\left.<36.2^{\circ} \mathrm{C}\right)$ twice in 8 hours; lethargy or hypotonia; feeding intolerance (feedings held for $>24$ h) in an infant tolerant of advancing or full feeds for 3 days; immature/total neutrophil (I:T) ratio $>0.2$; white blood cell count $>25,000$ or $<5,000 / \mathrm{mm}^{3}$; hyperglycemia $(>180 \mathrm{mg} / \mathrm{dL})$.

Other findings that were considered but did not remain sufficiently associated with sepsis after taking all the other findings into account were: New onset thrombocytopenia $\left(<100,000 / \mathrm{mm}^{3}\right)$; severe hypotension requiring volume or pharmacologic support; increased support on nasal CPAP, nasal cannula or hood oxygen.

We assigned clinical illness points a duration of 12 hours or until a follow-up normal laboratory result or a change in the type of ventilatory support occurred.

Statistical analysis. We used multivariable logistic regression modeling adjusted for repeated measures and a Wald $\chi^{2}$ test of the hypothesis that variables added independent information $(16,17)$. Internal validation of the predictive logistic regression models was performed using a bootstrapping methodology that has been recommended as more efficient over traditional split-sample approaches (22). Predictions for each infant were calculated based on coefficients estimated using a training set of all other infants. We evaluated the correlation of HRC measures with clinical signs using bootstrapped estimates of the $p$-value. Confidence intervals for the correlation coefficients, also determined by bootstrap, were $<0.03$.

\section{RESULTS}

Clinical and HRC database. Demographics of the patient population are shown in Table 1 . We prospectively recorded

Table 1. Demographics of the study population, and episodes of acute neonatal illness

\begin{tabular}{lcc}
\hline & Total & $<1500 \mathrm{~g}$ \\
\hline No. of infants & 337 & 172 \\
Birth weight & $1,460(910,2,539)$ & $915(715,1,177)$ \\
Gestational age & $30(27,35)$ & $27(25,28)$ \\
Male (\%) & 56 & 55 \\
Illness events & & \\
$\quad$ Positive blood culture (infants) & $76(63)$ & $67(55)$ \\
$\quad$ Negative blood culture (infants) & $80(63)$ & $67(53)$ \\
\hline
\end{tabular}

Results are presented as median $\left(25^{\text {th }}, 75^{\text {th }}\right.$ percentiles) or episodes (patients). more than 57,000 clinical observations in 337 infants. There were 76 episodes of proven sepsis and 80 episodes of clinical sepsis. As expected, the majority of cases of sepsis occurred in VLBW infants.

Descriptions of illustrative cases. Figure 1 shows graphical displays of clinical data and HRC index for the hospital courses of three infants. The top half of each panel shows the presence of selected clinical signs, and the shade of the bar reflects the severity of the sign and the clinical point score associated with it. The bars representing blood cultures have duration equal to the antibiotic therapy. The bottom half shows the HRC index (solid line) and the clinical score (dashed line).

Figure $1 A$ shows the benign course of an infant born at gestational age $29 \mathrm{wk}$ and birth weight $1,149 \mathrm{~g}$. After the first several days of life, there were no major clinical events or major HRC abnormalities during the nearly 8 wk course. The infant was intubated for about $1 \mathrm{wk}$ and on other oxygen support until day 45. Blood and urine cultures were drawn late on day 20 because of increasingly frequent episodes of apnea followed by temperature instability. Laboratory results of WBC, I:T ratio, platelet count blood glucose were normal. Cultures were negative, and antibiotics were administered for $2 \mathrm{~d}$.

Figure $1 B$ shows the course of an infant of gestational age 29 wk and birth weight $1,285 \mathrm{~g}$ who had an episode of late-onset neonatal sepsis. On day 18, Klebsiella sepsis was diagnosed and manifest by feeding intolerance and need for intubation and mechanical ventilation. On day 21 , he failed extubation and required re-intubation. On day 40, there was an increase in the clinical score due to temperature instability, frequent apnea and feeding intolerance, but this was not accompanied by a rise in HRC index, positive blood culture, prolonged antibiotic therapy or abnormal laboratory tests.

Panel $\mathrm{C}$ shows the course of another infant, gestational age 26 wk and birth weight $715 \mathrm{~g}$, who had an episode of late-onset neonatal sepsis. On day 30 there was an episode of Klebsiella sepsis with hyperglycemia and need for intubation and mechanical ventilation. On day 107, an abrupt rise and fall of the HRC index marks the time of bilateral inguinal herniorrhaphy and circumcision under anesthesia.

From inspection of graphical records of individual patients, we hypothesized that both HRC and clinical signs report on neonatal sepsis. If the hypothesis is true, then HRC and clinical illness should rise before the clinical diagnosis, HRC and at least some clinical signs should be correlated, and HRC and clinical signs should predict sepsis.

A clinical score for neonatal sepsis. The performance of predictive models depended on the time window of the training set. Clinical signs are the usual means for suspecting the diagnosis of neonatal sepsis, and are thus clustered around episodes of illness. If the time window for model-training included the time of diagnosis (that is, the time of the blood culture), model performance was improved. If the time window was just before, but did not include the time of the blood culture, signs were sparse and model performance deteriorated. In either case, there was highly significant association with feeding intolerance, increased frequency and severity of 

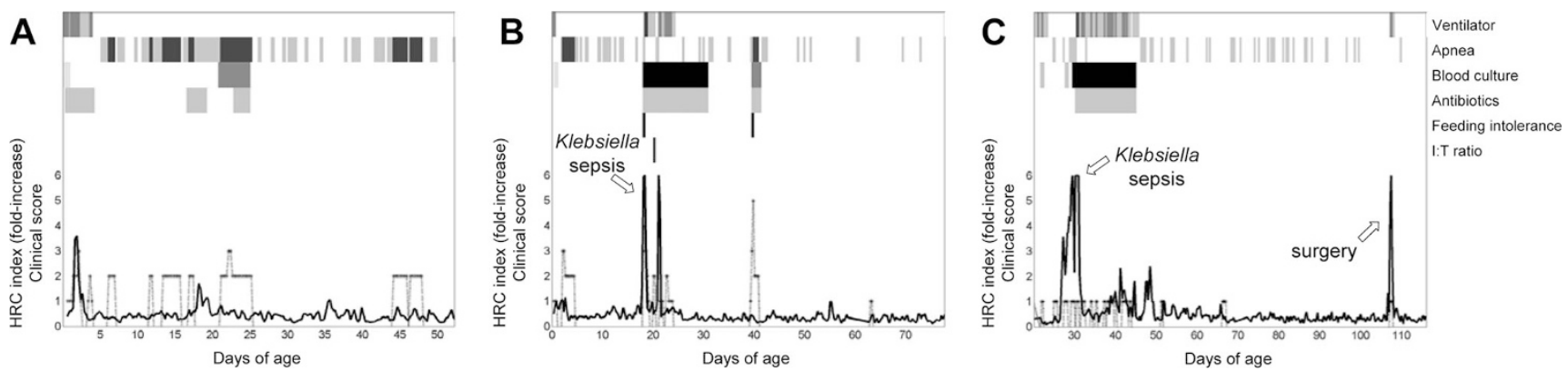

Figure 1. Graphical representations of the hospital course of three infants. In the upper half of each panel, the bars represent clinical findings listed on the right axis, and darker shading reflects more severely abnormal findings and higher clinical point scores (light gray 0 points, gray 1 point, black 2 points). In the bottom half, the noisy line is the HRC index and the dashed lines are the clinical score.

apnea, abnormal I:T ratio, need for increased support on mechanical ventilator, and temperature instability with the diagnosis of sepsis. Hyperglycemia and abnormal WBC were significantly associated with sepsis only when the time window included the time of the blood culture. Conversely, the finding of hypotonia and lethargy was significantly associated with sepsis only when the time window preceded the blood culture. Based on the regression coefficients, we assigned the point score shown in Table 2. Systematic analysis of changing each coefficient confirmed that the final score was optimal.

Several clinical findings commonly associated with sepsis did not appear in the final score. Severe hypotension is certainly a sign of sepsis, but occurred very infrequently in this population - only $3 \%$ of infants with sepsis had this sign. Thus it failed to reach sufficient significance in our scheme to warrant a point. New-onset thrombocytopenia is also a finding in sepsis, but did not reach sufficient association with sepsis after taking everything else into account.

HRC index and clinical score increase at the time of neonatal sepsis. Figure 2 shows the mean HRC index and clinical scores as a function of time relative to the 156 episodes of proven or clinical neonatal sepsis. The plots have been scaled to allow comparison of the changes near the time of sepsis. Asterisks mark data points that differed significantly from $24 \mathrm{~h}$ prior $(p<0.05, t$-test). The HRC index rises first, and shows significant increases over the $24 \mathrm{~h}$ before clinical suspicion.

Table 2. Point score for imminent neonatal sepsis

\begin{tabular}{lc}
\hline \multicolumn{1}{c}{ Signs } & Points \\
\hline Feeding intolerance (feedings held for greater than 24 & 2 \\
hours) in an infant who had been tolerating advancing & \\
or full feeds for three days & 2 \\
Severe apnea requiring positive pressure ventilation & 2 \\
$50 \%$ increase in number of apneic episodes over a 24 & \\
$\quad$ hour period in an infant who had been extubated and & \\
stable for three days & 2 \\
Immature/total neutrophil (I:T) ratio greater than 0.2 & 1 \\
Increase in ventilatory support and $\mathrm{F}_{\mathrm{i}} \mathrm{O}_{2}$ by $25 \%$ from & 1 \\
baseline & 1 \\
Lethargy or hypotonia & \\
Temperature instability ( $>38^{\circ} \mathrm{C}$ or $\left.<36.2^{\circ} \mathrm{C}\right) ;$ two & 1 \\
episodes within an eight hour period & 1 \\
Hyperglycemia $(>180 \mathrm{mg} / \mathrm{dL})$ & \\
Abnormal white blood cell count $(>25,000$ or $<5,000)$ & \\
\hline
\end{tabular}

HRC correlates with clinical signs of sepsis. The mean HRC index, taken over the entire hospital course for each infant, was highly significantly correlated with the mean clinical score $(r=0.55, n=337$ infants, $p<0.001)$. In addition, the 6-hourly HRC index measurements $(n=41,769)$ were highly significantly associated with the corresponding 6-hourly clinical scores, with correlation coefficient $r=0.27$ (95\% confidence interval $0.24-0.30$ ). Each of the candidate signs was significantly correlated with the HRC index $(p<$ $0.01)$. The highest correlation was with increased ventilatory support, with $r=0.24$ (95\% confidence intervals 0.21-0.27). Correlations with the laboratory tests led to $r$ values between 0.14 and 0.16 , and correlations with the other signs led to $r$ values between 0.04 and 0.07 .

HRC and clinical scores predict sepsis. Individually, both the clinical score and the HRC index were predictive of sepsis in the next $24 \mathrm{~h}$ (ROC areas 0.62 and 0.67 , respectively, $p<$ 0.001). Knowledge of both led to higher association with imminent sepsis (ROC area $0.70, p<0.001$ ), and the HRC index added significantly to the clinical score $(p<0.001)$. The performance of models that included data from the time of diagnosis was better, with ROC areas of 0.80 (clinical score),

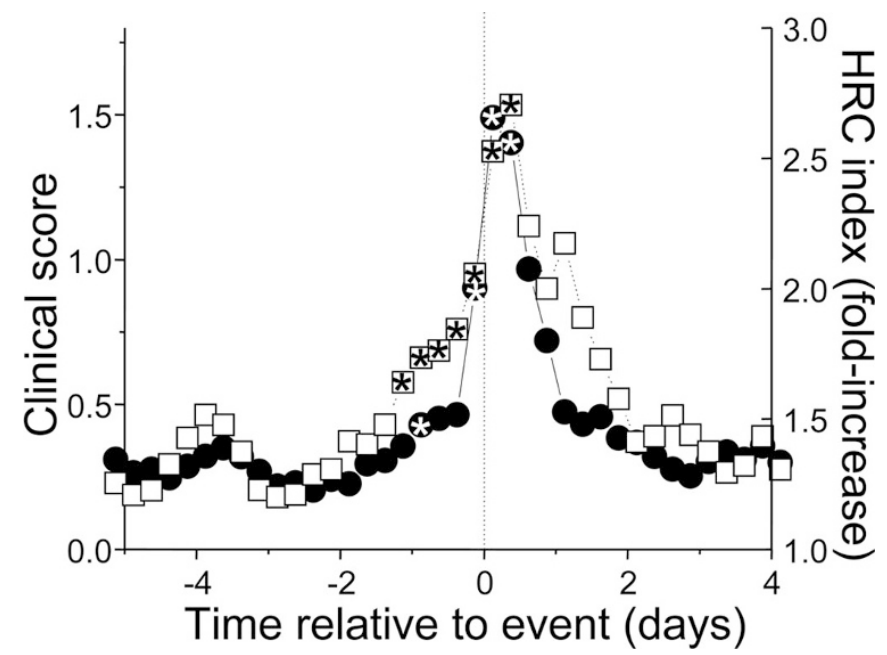

Figure 2. Changes in clinical score (๑) and HRC index ( $\square$ ) in relation to episodes of neonatal sepsis. Asterisks mark points that were statistically significantly increased compared with $24 \mathrm{~h}$ prior. The plots are scaled to have the same baseline and peak to allow comparison of the changes before the clinical illness. 


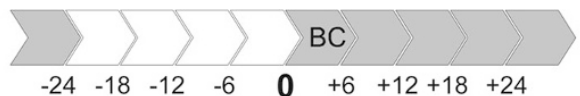

HRC - high-risk

HRC - low-risk

feeding intolerance

hypotonia

I:T ratio $>0.2$

apnea

increased ventilator

temp instability

hyperglycemia

abnormal WBC

0.1

1

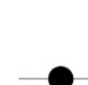

1
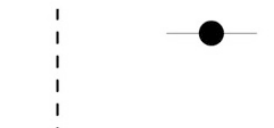

1
1
1
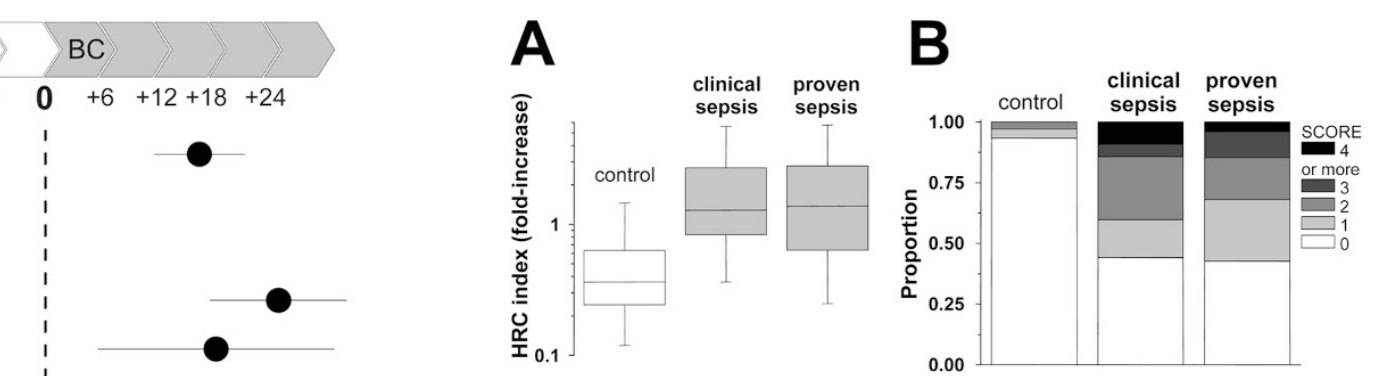

Figure 5. HRC index measurements in the $6 \mathrm{~h}$ before the diagnosis of clinical sepsis ( $n=77$ of 80 episodes) or proven sepsis (75/76) compared with control measurements $(n=211)$. B. Clinical scores. Neither HRC measurements nor clinical scores differ between clinical and proven sepsis, though both are highly significantly higher than control.

HRC index in diagnosing the symptomatic infant. The major finding is that clinical signs and tests are less useful in the time window preceding sepsis, because they are present much less often. This is expected - once findings are present, we expect the diagnosis to be suspected and tested for promptly. Here we categorized the HRC index into low: 70\% lowest values, less than 1-fold increase in risk; intermediate: $70^{\text {th }}$ to $90^{\text {th }}$ percentile values, 1- to 2-fold increase (not shown); and high-risk: $10 \%$ highest values, more than 2 -fold increase groups (19). After taking all other information into account, feeding intolerance was the most predictive clinical sign of neonatal sepsis.

Similarity of HRC and clinical signs in clinical and proven neonatal sepsis. Figure $5 A$ shows box plots of the HRC index for the 6-h period just before the diagnosis of clinical sepsis or proven sepsis. Control values were chosen 1 wk before event, or randomly from infants who did not have events. The HRC index values before proven or clinical sepsis were significantly different from control, but not from each other.

Figure $5 B$ shows bar graphs of clinical scores for the same infants. Infants with clinical or proven sepsis had higher scores than controls, but were not distinguishable from each other. More detailed inspection of individual clinical signs failed to reveal distinctive differences between infants with clinical and proven sepsis (not shown).

A risk assessment tool for early diagnosis of imminent neonatal sepsis. Figure 6 shows a table for bedside use in estimating the risk of impending sepsis in the NICU using a

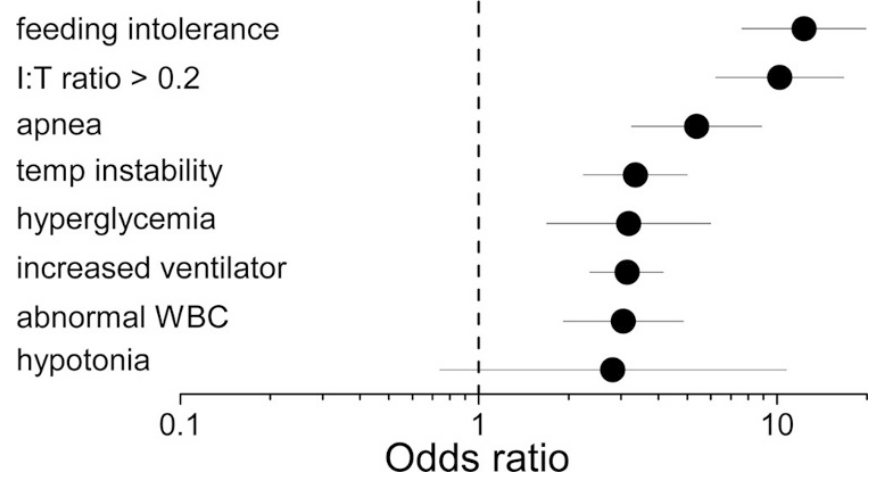

Figure 4. Odds ratios for clinical findings and the HRC index at the time of diagnosis of clinical and proven sepsis. Horizontal lines are 95\% confidence limits. The inset above shows the time window for training the models - the $6 \mathrm{~h}$ preceding and $18 \mathrm{~h}$ following the time of the blood culture obtained for suspicion of sepsis (BC).

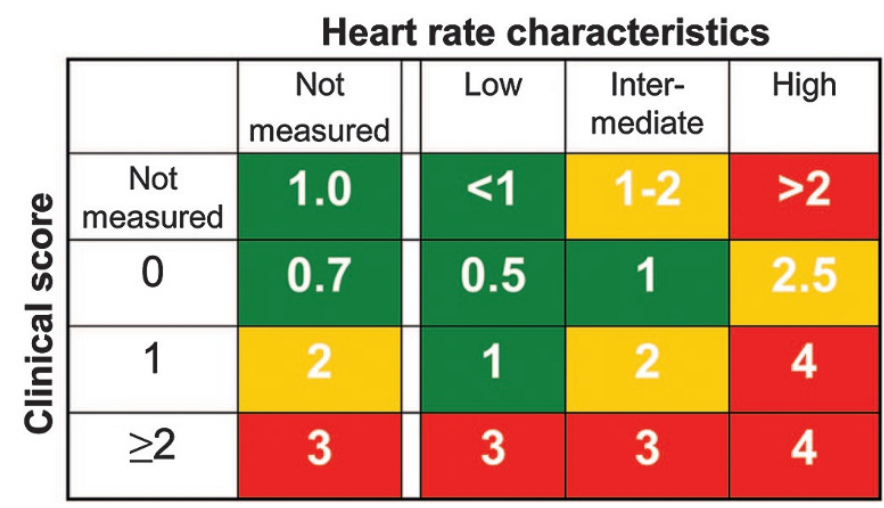

Figure 6. A risk assessment card for neonatal sepsis based on clinical signs and HRC monitoring. 
clinical score and HRC monitoring. When neither is measured, the fold-increase in risk of illness is 1.0. The clinical score alone differentiates infants across a spectrum of risk, as does the HRC index. Knowledge of both allows greater refinement in the estimation of risk. For example, an infant with a clinical score of 0 generally has lower risk of illness, but the concurrent finding of a high-risk HRC index identifies a subset with 2.5-fold increase in risk. For infants with 2 points or more, that is, with clinical findings of illness, knowledge of the HRC index adds little and even a low-risk HRC index does not cancel out the clinical presentation. This is in keeping with the idea that the HRC index is adjunctive to clinical information, and is not a standalone substitute for medical personnel.

\section{DISCUSSION}

We studied heart rate characteristics (HRC) and objective findings of clinical illness in infants in a tertiary care NICU. The major findings were (1) a validated HRC index was correlated with clinical signs of illness and abnormal laboratory tests as well as a composite score of clinical illness severity, and (2) the HRC index added significantly to clinical signs and abnormal laboratory tests in predicting imminent sepsis.

The mechanism by which sepsis leads to reduced variability and transient decelerations of heart rate is not known, though we speculate that circulating cytokines may interfere with normal signal transduction in sinus node pacemaker cells. There is substantial evidence that sepsis causes large increases in the circulating levels of many cytokines (24), and that these changes are evident before the clinical diagnosis of illness in newborn infants (25). Sepsis is not the only illness in the NICU that alters cytokine levels, and we know that the HRC index also increases with illnesses such as necrotizing enterocolitis, intracranial hemorrhage and respiratory failure, and at the time of surgery. Sepsis remains the focus of our work with HRC monitoring as it is common, and it seems reasonable that earlier diagnosis and therapy might improve outcome.

Inclusion of infants with negative blood cultures in studies of neonatal sepsis. Stoll et al. (9) in the NICHD Neonatal Research Network recently reported on neurodevelopmental impairment (NDI) in extremely low birth weight $(<1000 \mathrm{~g}$, ELBW) infants. The major finding was that infants who had one or more episodes of infection had a $50 \%$ increase in the already high rate of NDI. Importantly, NDI was equally increased in infants with "clinical sepsis," that is, sepsis-like illness warranting five or more days of antibiotics despite negative blood cultures. This finding justifies inclusion of these patients in evaluating novel means for early diagnosis of neonatal sepsis, and it is reasonable to suspect that some of them had bacterial sepsis that was not detected by blood culture, an imperfect test. Certainly the NICHD Neonatal Research Network clinicians must have believed the clinical presentation more than the blood culture, as prolonged courses of antibiotics were administered. Our findings of similar HRC and clinical signs in proven and clinical sepsis are consistent with our earlier work $(11,16)$.
A clinical illness score relevant to neonatal sepsis. Neonatal sepsis is suspected because of clinical signs of illness. When signs are severe enough, prolonged antibiotic therapy is administered even if the blood culture is negative; this is "clinical sepsis." Thus using a clinical score to predict neonatal sepsis is circular - since the signs are the diagnosis, they will never fail as a diagnostic test. Laboratory tests are also problematic, as they are much more frequently available near the time of sepsis (19). A more stringent test of the utility of clinical signs in early diagnosis is to analyze them before the clinical diagnosis of sepsis, when subtle signs might be attributed to benign causes. This is the approach we used in developing the HRC index, where the outcome of interest was the $24 \mathrm{~h}$ before, but not including, the quarter-day in which the diagnosis was made.

Here we developed and validated internally a clinical score following a method similar to that used by Escobar and coworkers to produce the Richardson score for neonatal mortality or prolonged ventilation (20). We began with clinical signs that we knew were associated with sepsis, and optimized their weighting. As expected, these signs were more predictive of sepsis when observed at the time of diagnosis. There were, however, highly significant associations in the preclinical phase as well. The most predictive findings were feeding intolerance, hypotonia and lethargy, abnormal I:T ratio, severe or increased apnea, need for increased support on mechanical ventilator, and temperature instability. The finding that I:T ratio was the most robust independent predictor of sepsis confirms our recent finding in a superset of this population (19).

We found a small increase in the clinical score in the $24 \mathrm{~h}$ before the clinical diagnosis of illness. This finding, which further validates the score, is not surprising since sepsis and SIRS are preceded by a period during which cytokines are elevated though symptoms are subtle or absent (25). This finding confirms that of Fanaroff et al. (2) that clinical signs were increased before the diagnosis of proven sepsis, and extends it by combining them into a clinical illness severity score and demonstrating similar changes before clinical sepsis. However, clinical experience teaches that the early diagnosis of neonatal sepsis and SIRS using clinical signs and laboratory findings alone is unsatisfactory, and that new diagnostic measures are badly needed $(1,26)$.

Surprisingly, up to $40 \%$ of infants with clinical and proven sepsis had 0 points. Several factors could contribute to this finding. First, several of our signs required the presence of an abnormal clinical sign or lab test for 8 or $24 \mathrm{~h}$. Thus a very acute illness would not score points. Second, infants might have had values near but not quite exceeding the threshold for points. For example, an infant with a $40 \%$ increase in apnea, $18 \mathrm{~h}$ of feeding intolerance, one episode of temperature instability and an I:T ratio of 0.18 would have 0 points despite an overall clinical presentation that could represent impending sepsis. Different thresholds and different tests would lead to different results, but scoring systems with hard cutoffs all suffer this shortcoming.

HRC index is correlated with clinical signs of illness and abnormal laboratory tests. We found that the HRC index was highly significantly associated with objective clinical findings 
of illness and with abnormal test results. There were correlations with individual signs and tests, and with the composite clinical score whether measured at each $6 \mathrm{~h}$ time point or averaged over the entire hospital course. Correlations were moderate or weak in degree.

Summary. In summary, we have devised an internally validated point score system relevant to late-onset neonatal sepsis. We found that heart rate characteristics (HRC) monitoring is correlated with this score, adds independent information to it, and becomes abnormal before it, among infants with clinical and proven neonatal sepsis. Since the HRC index is continuous and in real time, it does not require any new contact with the patient, and is significantly associated with imminent clinical neonatal illness, we feel that it may have a favorable impact on clinical care. While it will not replace clinical assessment by the physician, blood cultures or other laboratory tests, HRC monitoring can add information to conventional measures in the early diagnosis of neonatal sepsis.

\section{REFERENCES}

1. Stoll BJ, Hansen N, Fanaroff AA, Wright LL, Carlo WA, Ehrenkranz RA, Lemons JA, Donovan EF, Stark AR, Tyson JE, Oh W, Bauer CR, Korones SB, Shankaran S, Laptook AR, Stevenson DK, Papile LA, Poole WK 2002 Late-onset sepsis in very low birth weight neonates: the experience of the NICHD Neonatal Research Network. Pediatrics 110:285-291

2. Fanaroff AA, Korones SB, Wright LL, Verter J, Poland RL, Bauer CR, Tyson JE, Philips JB, Edwards W, Lucey JF, Catz CS, Shankaran S, Oh W 1998 Incidence, presenting features, risk factors and significance of late onset septicemia in very low birth weight infants. The National Institute of Child Health and Human Development Neonatal Research Network. Pediatr Infect Dis J 17:593-598

3. Kellogg JA, Ferrentino FL, Goodstein MH, Liss J, Shapiro SL, Bankert DA 1997 Frequency of low level bacteremia in infants from birth to two months of age. Pediatr Infect Dis J 16:381-385

4. Schelonka RL, Chai MK, Yoder BA, Hensley D, Brockett RM, Ascher DP 1996 Volume of blood required to detect common neonatal pathogens. J Pediatr 129:275278

5. Neal PR, Kleiman MB, Reynolds JK, Allen SD, Lemons JA, Yu PL 1986 Volume of blood submitted for culture from neonates. J Clin Microbiol 24:353-356

6. Pierce JR, Merenstein GB, Stocker JT 1984 Immediate postmortem cultures in an intensive care nursery. Pediatr Infect Dis 3:510-513

7. Squire E, Favara B, Todd J 1979 Diagnosis of neonatal bacterial infection: hematologic and pathologic findings in fatal and nonfatal cases. Pediatrics 64:60-64
8. Garner JS, Jarvis WR, Emori TG, Horan TC, Hughes JM 1988 CDC definitions for nosocomial infections, 1988. Am J Infect Control 16:128-140

9. Stoll BJ, Hansen NI, Adams-Chapman I, Fanaroff AA, Hintz SR, Vohr B, Higgins RD 2004 Neurodevelopmental and growth impairment among extremely low-birthweight infants with neonatal infection. JAMA 292:2357-2365

10. American College of Chest Physicians/Society of Critical Care Medicine Consensus Conference Committee (ACCP/SCCM) 1992 American College of Chest Physicians/Society of Critical Care Medicine Consensus Conference: Definitions for sepsis and organ failure and guidelines for the use of innovative therapies in sepsis. Crit Care Med 20:864-874

11. Griffin MP, Moorman JR 2001 Toward the early diagnosis of neonatal sepsis and sepsis-like illness using novel heart rate analysis. Pediatrics 107:97-104

12. Moorman JR, Lake DE, Griffin MP 20065 Heart rate characteristics monitoring in neonatal sepsis. IEEE Trans Biomed Eng 53:126-132

13. Kovatchev BP, Farhy LS, Cao H, Griffin MP, Lake DE, Moorman JR 2003 Sample asymmetry analysis of heart rate characteristics with application to neonatal sepsis and systemic inflammatory response syndrome. Pediatr Res 54: 892-898

14. Lake DE, Richman JS, Griffin MP, Moorman JR 2002 Sample entropy analysis of neonatal heart rate variability. Am J Physiol Regul Integr Comp Physiol.Am J Physiol 283:R789-R797

15. Richman JS, Moorman JR 2000 Physiological time series analysis using approximate entropy and sample entropy. Am J Physiol Heart Circ Physiol.Am J Physiol 278:H2039-H2049

16. Griffin MP, O'Shea TM, Bissonette EA, Harrell FE Lake DE, Moorman JR 2003 Abnormal heart rate characteristics preceding neonatal sepsis and sepsis-like illness. Pediatr Res 53:920-926

17. Griffin MP, O'Shea TM, Bissonette EA, Harrell FE Jr, Lake DE, Moorman JR 2004 Abnormal heart rate characteristics are associated with neonatal mortality. Pediatr Res 55:782-788

18. Griffin MP, Lake DE, Bissonette EA, Harrell FE Jr, O'Shea TM, Moorman JR 2005 Heart rate characteristics: novel physiomarkers to predict neonatal infection and death. Pediatrics 116:1070-1074

19. Griffin MP, Lake DE, Moorman JR 2005 Heart rate characteristics and laboratory tests in neonatal sepsis. Pediatrics 115:937-941

20. Escobar GJ, Shaheen SM, Breed EM, Botas C, Greene JD, Yoshida CK, Zupancic J, Newman TB 2004 Richardson score predicts short-term adverse respiratory outcomes in newborns $<=34$ weeks gestation. J Pediatr 145:754-760

21. Richman JS, Lake DE, Moorman JR Sample entropy. Methods Enzymol 2004;384:172-184

22. Steyerberg EW, Harrell FE, Borsboom GJ, Eijkemans MJ, Vergouwe Y, Habbema JD 2001 Internal validation of predictive models: Efficiency of some procedures for logistic regression analysis. J Clin Epidemiol 54:774-781

23. Moons KG, Harrell FE, Steyerberg EW 2002 Should scoring rules be based on odds ratios or regression coefficients? J Clin Epidemiol 55:1054-1055

24. Anderson MR, Blumer JL 1997 Advances in the therapy for sepsis in children. Pediatr Clin North Am 44:179-205

25. Kuster H, Weiss M, Willeitner AE, Detlefsen S, Jeremias I, Zbojan J, Geiger R, Lipowsky G, Simbruner G 1998 Interleukin-1 receptor antagonist and interleukin-6 for early diagnosis of neonatal sepsis 2 days before clinical manifestation. Lancet 352:1271-1277

26. Stoll BJ, Gordon T, Korones SB, Shankaran S, Tyson JE, Bauer CR, Fanaroff AA, Lemons JA, Donovan EF, Oh W, Stevenson DK, Ehrenkranz RA, Papile LA, Verter J, Wright LL 1996 Late-onset sepsis in very low birth weight neonates: a report from the National Institute of Child Health and Human Development Neonatal Research Network. J Pediatr 129:63-71 